QVIPURAMAYOC Revista de la Facultad de Ciencias Contables

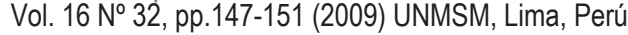

ISSN: 1560-9103 (versión impresa) / ISSN: 1609-8196 (versión electrónica)

\title{
LA INTERNACIONALIZACIÓN DE LA I+D COMO VENTAJA COMPETITIVA DE LAS EMPRESAS Y EL EMPRENDIZAJE
}

\author{
THE INTERNATIONALIZATION OF THE I+D AS ADVANTAGE \\ COMPETITIVE OF THE COMPANIES AND THE ENTERPRISING
}

\section{JeAN P. SeClen LunA*}

[Recepción: Abril de 2009 / Conformidad: Mayo de 2009]

\begin{abstract}
RESUMEN
Adquiriendo e integrando conocimientos procedentes de ubicaciones geográficamente dispersas, las empresas pueden generar una mayor innovación, de más valor y de menor coste. Sin embargo, la relación entre la diversidad geográfica y la del conocimiento es importante, pero no siempre es inmediatamente obvia. Por tanto, hallar nuevos nichos de conocimiento de valor con el fin de impulsar la innovación es parecido a realizar prospecciones en busca de oro. Por otra parte, el emprendedor juega un papel muy importante en el desarrollo de una región o país.
\end{abstract}

Palabras clave: Investigación y desarrollo, conocimiento, internacionalización y emprendizaje.

\begin{abstract}
Acquiring and integrating knowledge from geographically dispersed locations, companies can generate more innovation, more value and lower cost. However, the relationship between diversity and geographical knowledge is important, but not always immediately obvious. Therefore, finding new niches of knowledge of value in order to foster innovation is similar to prospecting for gold. Moreover, the entrepreneur plays a major role in the development of a region or country.

Keywords: Research and Development, Knowledge, Internationalization and Entrepreneurship.
\end{abstract}

\footnotetext{
MBA en Dirección de Empresas. Facultad de Empresariales de la Universidad de Mondragón. Diciembre de 2005.
} 


\section{1. ¿ES LA I+D SÓLO UN PROCESO ENDÓGENO?}

En un mundo que se está moviendo en nanosegundos, la globalización está conduciendo y replanteando las decisiones más importantes, definiendo las prioridades y haciendo que las inversiones estratégicas estén más cerca a los clientes, a los competidores y a los cambios tecnológicos. Actualmente, las empresas están reconociendo que el ser global no solamente es incrementar mercados en el exterior, sino también tener acceso a los recursos escasos, en especial, del conocimiento que se encuentra diseminado alrededor del mundo. Hasta hace poco, la mayoría del trabajo inteligente de las empresas ha estado concentrada en el mercado nacional, este hecho particularmente se da sobre la investigación y desarrollo. Sin embargo, la nueva tendencia es internacionalizar esta tarea, para ello, las empresas están a la caza de las habilidades y talentos para la $\mathrm{I}+\mathrm{D}+\mathrm{i}$, porque consiguen una mayor ventaja competitiva al innovar con menores costos, tener acceso a redes de investigación y mercados potenciales. No obstante, tienen que buscar el equilibrio a la pérdida de trabajos en su país de origen.

Por el contrario, los países receptores de estas inversiones fortalecen su capacidad de innovación aprovechándose de las redes internacionales que tienen las empresas transnacionales; sin embargo, el reto de estos países pasa por reunir las condiciones necesarias para atraer dicha inversión: Esto depende de la calidad de los recursos humanos y de las instituciones, así como de la capacidad de las empresas nacionales, contando para ello con parques científicos, laboratorios públicos de $\mathrm{I}+\mathrm{D}$, incubadoras de empresas, estableciendo sistemas de protección de la propiedad intelectual, tratando de equili- brar el control extranjero sobre sus recursos de $\mathrm{I}+\mathrm{D}$ como lo viene haciendo China, por ejemplo.

Por otro lado, el retiro de las barreras al comercio internacional ha creado las oportunidades para que las empresas se internacionalicen. Las multinacionales a través de sus mejores prácticas logran su sostenibilidad, por ello, es un paradigma a seguir mediante una reflexión estratégica. Actualmente, el flujo de inversiones en actividades de I+D cada vez es mayor en algunos países en desarrollo. En China, por ejemplo, el número de unidades extranjeras de $\mathrm{I}+\mathrm{D}$ pasó de ninguna a 700 en diez años. Antes se realizaban actividades de I + D para adaptar productos y procesos a las exigencias de los mercados locales, ahora se tiende al desarrollo tecnológico para mercados mundiales y a la investigación aplicada. A pesar de todo, la internacionalización de la I $+D$ ha sido un área casi ausente en la teoría económica y empresarial hasta la década de los ochenta, sin olvidar algunas contribuciones pioneras como de Cordell (1973) y Ronstadt (1977). Desde entonces existe una considerable literatura tratando de entender y explicar por qué las empresas acuden al exterior a realizar parte de sus investigaciones y cuáles son las pautas que caracterizan dicha expansión.

El marco teórico en el que se insertan los estudios sobre la localización de la actividad tecnológica ha ido cambiando a medida que evolucionaba el análisis económico de la innovación y el cambio tecnológico (Nelson y Winter, 1982), la acumulación tecnológica (Pavitt, 1987; Cantwell, 1989); el aprendizaje interactivo y social (Lundwall, 1988) y el enfoque de red (Aydalot, 1986; Osborn y Hagedoorn, 1997; Zander, 1999).

Las actividades de I+D no están supeditadas absolutamente al tamaño de las empresas, las PYME lo pueden hacer a través de outsourcing o alianzas para conseguir economías 
de escala, otra alternativa es la adquisición o el licenciar la tecnología existente en otros países. Por otro lado, hay empresas conservadoras que creen que la internacionalización de la $\mathrm{I}+\mathrm{D}$ es la última fase del proceso de internacionalización de una empresa, es decir, es después de la implantación de las filiales productivas en el exterior.

Sin embargo, hay otras empresas más efectivas que una vez que se han posicionado en su mercado local, deciden salir al exterior haciendo primero una buena labor de benchmark, segmentación de potenciales clientes y competidores para después acudir a los mejores del sector y desarrollarse a través de alianzas estratégicas. Así fue la experiencia de Shiseido que es una empresa de cosméticos de Japón que quiso competir en el mundo del perfume y después de segmentar su mercado objetivo, decidió aprender y competir poniendo su centro de $\mathrm{I}+\mathrm{D}$ en París. En consecuencia, para el desarrollo de la internacionalización de I+D no hay receta única. Entonces podemos definir que la internacionalización de la I+D no es sólo llevar los propios conocimientos a los nuevos mercados, sino capturar otros conocimientos. Por lo tanto, es localizar los centros de I+D en diferentes lugares del mundo, a fin de obtener significativas ventajas que aumenten el propio conocimiento.

Para finalizar, es necesario que los agentes públicos y privados desarrollen un gran esfuerzo por consolidar la innovación y el conocimiento como motores fundamentales del desarrollo económico, tecnológico y social. Es decir, debe generarse sinergias entre la universidad, empresa y gobierno. Por ello, los gobiernos deben hacer de la innovación el eje principal de su política económica, adaptando su modelo productivo y apostar por producciones de alto valor añadido en la búsqueda de tecnología propia en sectores tractores. En consecuencia, es imprescindible la existencia previa de un ambiente que haga atractiva la actividad empresarial y que premie generosamente la actividad innovadora de las empresas.

En definitiva, el reto que afrontan las empresas ante el futuro pasa por innovar con creatividad y deben apoyarse en la $\mathrm{I}+\mathrm{D}$, que al fin y al cabo, este gasto a priori diverge con las necesidades primordiales de las empresas, puesto que estas inversiones son a largo plazo. Sin embargo, la sostenibilidad de las empresas depende de dichas inversiones.

\section{2. ¿EL EMPRENDEDOR CONTRIBUYE A LA COMPETITIVIDAD DE UNA REGIÓN O PAÍS?}

Hay que ser conscientes de la realidad económica en que vivimos, estamos en una economía globalizada donde no podemos ser indiferentes y estar aislados. La economía mundial se dirige hacia un nuevo paradigma económico basado en el conocimiento que tiene un claro valor diferenciador. Los factores claves del desarrollo económico que pueden ser gestionados a voluntad de las personas y las sociedades son dos: el primero es el factor trabajo donde la formación de las personas es determinante (además se debe considerar que el valor añadido que cada individuo aporta a su trabajo depende de su motivación y de sus conocimientos), el segundo factor es el nivel tecnológico con que se cuenta (siendo éste del entorno o importado).

En la Unión Europea se realizan a menudo estudios y análisis donde el emprendizaje es una parte importante de dichos trabajos, por cuanto más del $80 \%$ del empleo actual se encuentra en las PYME y la tendencia futura del empleo se centra en el emprendedor local. Por otro lado, las PYME cuentan con 
importantes ventajas como agilidad de adaptación al cambio, cercanía al mercado local y capacidad de crecimiento. Por tanto, no se debe ver la creación de nuevas empresas motivadas por la falta de oportunidades de empleo o como pequeños negocios que no poseen un alto valor añadido que contribuyen de manera mínima al PIB, sino, como motor de crecimiento de la economía. Al no contar con infraestructuras o apoyos exógenos, las nuevas empresas están destinadas al fracaso. Por ejemplo, la cultura emprendedora de los Estados Unidos pone énfasis en los agentes claves centrándose en los emprendedores, inversores de capital y en políticas diseñadas para crear infraestructuras que promuevan la ventaja competitiva. En tal sentido, el gobierno juega un papel muy importante como promotor de la cultura emprendedora.

Es indispensable que para que el emprendedor se haga como tal, cuente con condiciones necesarias o entornos favorables. Por ejemplo, en Australia hacen falta dos días para dar de alta a una empresa con todos los permisos legales y administrativos, en cambio en Haití se necesitan 203 días. En consecuencia, podemos afirmar que la burocracia ralentiza o inhibe la competitividad e innovación en muchos países. Por ello, cuando las políticas estatales son bien diseñadas y aplicadas en todos los niveles pueden conducir a la innovación. Pero, ¿hasta qué punto las políticas estatales conducen a la innovación y promueven el emprendizaje? Las empresas, especialmente las pequeñas, están en el centro de la competitividad de cualquier economía. La facilidad o dificultad de poner o ampliar una empresa, de realizar operaciones diarias e incluso de cerrarla está determinada en gran medida por los trámites administrativos impuestos por los Estados. En los países que desean mejorar su competitividad económica es esencial hacer que los entornos operativos de las empresas sean lo más simple posible. Y facilitar que los empresarios creen empresas es tan importante como crear nuevos empleos para el crecimiento económico.

Otros promotores son las cámaras de comercio, cuyas funciones más significativas son las ayudas a la internacionalización, asesoría en temas de innovación, tecnología e información económica. Los centros de empresas innovadoras están dedicados a la promoción de proyectos empresariales innovadores, motivan a los emprendedores para el desarrollo de iniciativas, apoyan en la elaboración de informes de viabilidad, plan de negocios y subvenciones para la puesta en marcha del proyecto. Finalmente, la participación aislada del emprendedor en la economía de un país está destinada al fracaso. En consecuencia, es necesario para la sostenibilidad de los mismos, contar con un entorno favorable, siendo éste el reto a afrontar por los agentes claves que promocionan al emprendedor. Por otro lado, el reto del emprendedor es innovar. En definitiva, la creación de nuevas empresas innovadoras aporta a la creación de nuevos empleos, ingresos fiscales, equilibrio del tejido empresarial y al crecimiento de la economía.

\section{CONCLUSIONES}

$\mathrm{Al}$ internacionalizar la $\mathrm{I}+\mathrm{D}$, las empresas consiguen una mayor ventaja competitiva al innovar con menores costos, tener acceso a redes de investigación y mercados potenciales.

La IED en actividades de I+D beneficia a emisores y receptores. El reto pasa por buscar el equilibrio adecuado.

Actualmente, el flujo de inversiones en actividades de $\mathrm{I}+\mathrm{D}$ cada vez es mayor en algunos países en desarrollo. Antes se realizaban actividades de I+D para adaptar productos y procesos a las exigencias de los mer- 
cados locales. Ahora, se tiende al desarrollo tecnológico para mercados mundiales y a la investigación aplicada.

La creación de nuevas empresas innovadoras aportan a la creación de nuevos empleos, ingresos fiscales, equilibrio del tejido empresarial y al crecimiento de la economía.

\section{REFERENCIAS BIBLIOGRÁFICAS}

1. Aydalot, P.H (1986). Milieux innovateurs en Europe. Paris

2. Cantwell, J (1989). “Technological innovation and multinational", Oxford.

3. Cordell, A (1973). "Innovation and the multinational corporation".

4. Dodgson, M (2000). "The management of Technological Innovation", Oxford

5. Doz, Santos, Willliamson (2001). "From Global to metanacional"

6. Dunning, J y Narula (1995). "The R\&D activities of foreing firm in the USA"

7. Lundval, B (1988). "Technical change and economic theory", Londres

8. Mowery (1983). "The relationship between intra firm and contractual form of industrial research in American manufacturing"

9. Osborn, R (1997). "The institutionalisations and evolutionary dynamics of inter organizational alliances and networks"

10. Pavitt, K (1987). “International paten's of technological acumulation". Londres.
11.Rondtandt, R. (1977). "Research and delevoping abroad by multinational", New York

12. Sampon, R. (2004). "Maximización de la innovación en alianzas del R\&D”

13. Tilton, J. (1971). "International difusión of technology", Washington

14. Vengelers, R. (1997). "Internal R\&D expenditures and external technology soursing"

15. Revista Innovación Europea, Comisión Europea, 2005

16. Revista Fomento a la innovación tecnológica, 2005

17. Revista Perspectivas CDTI, No 25, 2005

18. Review Harvard Business School, vol. 81,2003

19. The economist, Economist Intelligence Unit "World investment prospects, the revival of globalization”, 2004

20. OECD, 2004 "Globalizsation of R\&D, an OECD perspective

21. UNCTAD, World Investment report 2005, Transnational corporations and the internationalization of R\&D.

22. Plan Nacional de I+D+I, Ministerio de Ciencia y Tecnología, 2003

23. Libro Blanco del Sistema Vasco de Innovación, 2005

24. Informe COTEC 2005 "Tecnología e innovación en España”, 2005 